Pétrarque et le pétrarquisme

\title{
Le « pauvre Pétrarque » dans la réflexion poétique
} de France Prešeren

Contribution à l'histoire du pétrarquisme dans les littératures slaves

Irena Prosenc Šegula

\section{(2) OpenEdition}

Journals

Édition électronique

URL : http://journals.openedition.org/cei/620

DOI : $10.4000 /$ cei.620

ISSN : 2260-779X

Éditeur

UGA Éditions/Université Grenoble Alpes

Édition imprimée

Date de publication : 15 avril 2006

Pagination : 117-127

ISBN : 978-2-84310-081-9

ISSN : $1770-9571$

Référence électronique

Irena Prosenc Šegula, «Le « pauvre Pétrarque » dans la réflexion poétique de France Prešeren », Cahiers d'études italiennes [En ligne], 4 | 2006, mis en ligne le 15 octobre 2007, consulté le 30 avril 2019. URL : http://journals.openedition.org/cei/620 ; DOI : 10.4000/cei.620 


\title{
LE «PAUVRE PÉTRARQUE» DANS LA RÉFLEXION POÉTIQUE DE FRANCE PREŠEREN
}

\author{
CONTRIBUTION À L'HISTOIRE DU PÉTRARQUISME \\ DANS LES LITTÉRATURES SLAVES
}

\author{
Irena Prosenc Šegula \\ Université de Ljubljana
}

Cupido! tu e la tua bella vecchia,
più non mi prenderete per il naso;
gratis non canterò le vostre lodi
per sempre, come il povero Petrarca.

Dans la littérature slovène, l'influence de Pétrarque se fait sentir relativement tard par rapport aux autres littératures européennes. A l'exception de quelques rares mentions et citations qui paraissent dans les textes slovènes à partir du XVI e siècle, la poésie de Pétrarque n'entre dans l'espace culturel slovène que dans la première moitié du XIX siècle, avec le poète romantique France Prešeren.

Cette réception tardive (mais pas pour autant moins fructueuse) s'explique sur le fond de la lente évolution de la littérature slovène qui ne produit ses premières œuvres qu'autour de 1550 (notamment le Catechismus et l'Abecedarium de Primož Trubar, expression du mouvement protestant). Jusqu'au XIX siècle, la littérature slovène semble toujours être en retard par rapport aux principaux mouvements littéraires européens. Ce retard est étroitement lié à la situation politique: n'étant pas constituée comme nation proprement dite, la Slovénie se trouve sous l'influence politique et culturelle des pays voisins, ce qui comporte une forte prédominance de la langue allemande dans la vie publique. De nombreux auteurs slovènes écrivent, en effet, une partie de leurs œuvres en allemand, qui est aussi la langue par excellence de la correspondance, même privée. 
Pour la réception quelque peu tardive de Pétrarque en Slovénie, il est d'ailleurs significatif que la grande absente du développement littéraire slovène soit, justement, la Renaissance. Comme à ses débuts au XVI siècle, la littérature slovène continue à avoir comme domaines prépondérants les écrits religieux et les questions grammaticales et lexicales; cet état des choses ne change qu'à l'époque des Lumières, dans la seconde moitié du $\mathrm{XVIII}^{\mathrm{e}}$, et pendant les premières décennies du XIX siècle. Surgit alors une ambition de renouveler la littérature, que l'on continue toutefois à concevoir d'une manière nettement didactique, pragmatique, les lettres slovènes restant ainsi orientées vers des contenus, des formes et un langage "paysans", conformément à un topos très répandu qui considère des Slovènes comme de "paisibles paysans" ${ }^{1}$.

Pendant les siècles qui précèdent l'essor du courant romantique en Slovénie, Pétrarque ne reste cependant pas inconnu dans l'espace culturel slovène. Des éditions incunables de ses œuvres latines et italiennes sont conservées jusqu'à aujourd'hui, même s'il est, évidemment, difficile de dater avec certitude le moment de leur arrivée en Slovénie ${ }^{2}$. Il faudra toutefois attendre le $\mathrm{XX}^{\mathrm{e}}$ siècle pour voir paraître les premières traductions en slovène de ses poèmes ${ }^{3}$.

Le moment où l'influence de Pétrarque se fait sentir dans la poésie slovène coïncide aussi (et ce n'est pas qu'une coïncidence purement chronologique) avec la pleine insertion du romantisme slovène dans les courants littéraires européens, insertion dans laquelle France Prešeren (1800-1849) a un rôle de la plus haute importance. Le rôle de Prešeren, «le premier et principal classique de la poésie slovène » ${ }^{4}$, est crucial à plusieurs niveaux:

1. Voir Janko Kos, Pregled slovenskega slovstva, Ljubljana, DZS, 1983; Boris PATERnU, "Položaj slovenskega pesništva", in IDEM, France Prešeren in njegovo pesniško delo, I, Ljubljana, Mladinska knjiga, 1976-1977, p. 51-61 ; Anton SLODNJAK, «France Prešeren in njegove poezije», in France PrešEren, Poezije doktorja Franceta Prešerna, ed. A. Slodnjak, Ljubljana, Prešernova družba, $2002^{15}$ (1946), p. 5-34.

2. Voir Jože POGAčNIK, «Slovenski romantizam i Petrarca", in Petrarca i petrarkizam u slavenskim zemljama. Radovi Međunarodnog simpozija/Petrarca e il petrarchismo nei paesi slavi. Atti del convegno internazionale. Dubrovnik, 6. - 9. XI. 1974, ed. Frano Čale, Zagreb-Dubrovnik, JAZU, 1978, p. 425-426.

3. Quelques traductions de poèmes et fragments de poèmes se trouvent dans Pavel GrošELJ, "Prešeren in Petrarka: leposlovnozgodovinska črtica», in Zbornik znanstvenih in poučnih spisov, 4 (1902), p. 23-61. Des éditions de poèmes choisis ont paru ensuite, notamment par Alojz GRADNIK (Soneti in kancone, Koper, Primorska založba, 1954) et Andrej CAPUDER (plusieurs éditions de 1980, 1987, 1995 et 1998, dont la plus complète Francesco PetrarCA, Soneti, Ljubljana, Mihelač, 1995). Cependant, il manque toujours une édition intégrale des Rerum vulgarium fragmenta en slovène.

4. Boris PAternu, «Prešernovo mesto v slovenski knjiševnosti», in France PrešERen, Pesmi in pisma, ed. B. Paternu, Ljubljana, DZS, 2000, p. 9. 
seul véritable poète romantique slovène, il promeut le développement de la littérature en slovène, travaille au perfectionnement de la langue littéraire et contribue à la création de l'identité culturelle slovène. En fait, le mouvement romantique slovène ne compte fondamentalement que deux représentants: Prešeren et son mentor Matija Čop, théoricien du romantisme slovène. Etant donné que la poésie de Prešeren ne peut être définie comme romantique qu'à partir de 1830 approximativement, et qu'en 1848 disparaît définitivement L'Abeille carniolienne (Krajnska Čbelica), revue littéraire qui publiait, entre autres, des poèmes de Prešeren, la durée $\mathrm{du}$ romantisme slovène ne s'étend que de 1830 à $1848^{5}$. Après s'être consacré à l'édition du recueil de ses poèmes en un volume, Poezije (Poèmes), publié en 1846 (mais portant la date de 1847), Prešeren arrête d'écrire. Matija Čop, pour sa part, publie, malgré ses connaissances étendues de la littérature européenne et son intérêt pour les questions théoriques, relativement peu ce que Prešeren ne tardera pas à lui reprocher ${ }^{6}$, et, au moment de sa mort prématurée en 1835 , à regretter ${ }^{7}$.

En dépit de la brève durée du mouvement romantique, c'est précisément à cette époque que se produisent des changements importants dans la littérature slovène. Si nous nous limitons à la réception de la poésie italienne, ce sont surtout Pétrarque, Dante et le Tasse qui sont lus, étudiés, recommandés et ainsi introduits dans l'espace culturel slovène. Pour ce qui est de la poésie de Pétrarque, Prešeren la connaît à travers plusieurs

5. Voir Janko Kos, Primerjalna zgodovina slovenske literature, Ljubljana, Mladinska knjiga, 2001, p. 105 suiv.; idem, Pregled slovenskega slovstva, cit., p. 93ss. Kos identifie comme troisième représentant du romantisme slovène Stanko Vraz, un "romantique plutôt potentiel qu'effectif» (Primerjalna zgodovina slovenske literature, cit., p. 106). Il s'agit d'un partisan du mouvement illyrien (promouvant, entre autres, l'usage du croate en tant que langue littéraire aussi de la part des slovènes) qui, après avoir fait ses débuts littéraires en slovène, opte pour l'usage du croate.

6. Voir l'épigramme Un bizarre putois, in France PREšEREN, L'ultime aimée: poèmes, trad. par Kolja MičEvič, [S. 1.], Editions Kolja Mičevič, 2001, p. 109 (Čudni dihur, in Poezije doktorja Franceta Prešerna, cit., p. 276). Tous les poèmes de Prešeren dans leur version originelle sont cités selon l'édition de Slodnjak, à l'exception de quelques poèmes en allemand. Pour ce qui est des traductions, la situation est plus complexe: étant donné qu'à l'heure actuelle il n'existe que des éditions fragmentaires en français, nous avons opté pour les traductions en italien d'après deux éditions qui, dans leur ensemble, sont les plus complètes: France PrEšErEn, Poesie, ed. Anton Slodnjak, trad. Francesco Husu, Trieste, Editoriale Stampa Triestina, 1976; France PREŠEREN, Poesie, ed. Marija Pirjevec, trad. Giorgio Depangher, Comune di Kranj, Trieste, Editoriale Stampa Triestina, 1998. Cependant, l'épigramme susmentionné ne figure dans aucune des éditions italiennes, ce qui est tout à fait révélateur du manque de traductions intégrales. Voir à ce sujet Marija PIRJEVEC, «La fortuna di Prešeren in Italia», Ricerche slavistiche 1 (47), 2003, p. 71-80.

7. Dem Andenken des Matthias Čop, in Poezije doktorja Franceta Prešerna, cit., p. 328; Matiju $\breve{C}$ opu, épitaphe, ibid., p. 281 (il n'existe pas de traduction); In memoria di Mattia Čop, in Poesie, trad. par F. Husu, cit., p. 83 (V spomin Matija Čopa, in Poezije doktorja Franceta Prešerna, cit., p. 125). 


\section{IRENA PROSENC ŠEGULA}

sources. Selon toute probabilité, il lit Pétrarque au cours de ses études ${ }^{8}$; il possède certainement une édition florentine des Rime de $1822^{9}$. Par ailleurs, sa connaissance de Pétrarque est filtrée par le truchement de Čop, avec qui il collabore étroitement; par son intermédiaire il connaît également les idées du romantisme allemand, surtout des frères Schlegel, que Čop propage dans ses écrits. Ce dernier théorise et favorise l'introduction, dans la littérature slovène, des formes poétiques romanes, particulièrement du sonnet, à travers un choix d'auteurs classiques qui se révèle typiquement romantique:

Dem krainischen Dichter steht die Wahl der metrischen Formen um so mehr frei, da wir keine eigentlich nationalen [...] besitzen. Warum sollte er da nicht diejenigen wählen, die als die schönsten unter den neueren [...] allgemein anerkannt sind, die südeuropäischen nämlich, und namentlich die des benachbarten Italieners, die selbst von jenen Völkern, deren Sprachen sich denselben bei weitem weniger fügen, als die des Krainers, den Deutschen, Engländern etc. mühsam nachgebildet werden? Diese Formen sind übrigens auch von andern Slawen schon vielfältig gebraucht worden. Eine der schönsten unter denselben, das Sonett (eine Lieblingsform mehrerer der größten neueren Dichter seit dem dreizehnten Jahrhunderte, eines Dante, Petrarca, Tasso, Lope de Vega, Camoens, Shakspeare [sic], Milton etc., in Deutschland von den Dichtern der schlesischen Schule: Opitz, Flemming etc. eingeführt, dann von Bürger und Schlegel erneuert und seitdem viel bearbeitet) wurde [...] von dem ersten polnischen Dichter Ad. Mickiewicz mit der ihm eigenen Meisterschaft behandelt [...] Von den Preschern'schen Sonetten scheinen uns mehrere, namentlich die in der Kr. Zhb. II. str. 24, III. str. 29 vorkommenden, in ihrer Art musterhaft zu seyn. ${ }^{10}$

8. Voir Janko Kos, Primerjalna zgodovina slovenske literature, p. 88.

9. Voir l'inventaire de succession daté du 9 mars 1849, reproduit dans Avgust ŽIGON, Zapuščinski akt Prešernov, Kranj, 1904, où les Rime figurent sur la liste des livres en possession du poète au moment de son décès (p. 30). Dans son commentaire, Žigon tient à souligner explicitement que Prešeren «avait son propre Pétrarque» (p. 8); on ignore le sort de ce livre.

10. Matija ČOP, «Krajnska Cbelica. Na svitlobo dal M. Kastelic. 1-3 bukvice. V' Ljubljani, 1830-1832. (Die krainische Biene, herausgegeben von M. Kasteliz, Laibach, 1830-1832, 3 Bändchen)", Illyrisches Blatt, le 16 février 1833: "Le poète slovène est d'autant plus libre dans le choix des formes métriques que nous ne possédons pas de véritable tradition nationale [...] dans ce domaine. Alors pourquoi ne choisirait-il pas parmi les formes modernes [...] celles qui sont universellement réputées être les plus belles, à savoir celles de l'Europe méridionale, notamment celles de l'Italie voisine, qu'imitent, parfois avec difficulté, même ces peuples, comme les Allemands et les Anglais, dont la langue s'y prête beaucoup moins que la nôtre. Ces formes ont d'ailleurs déjà été souvent utilisées par d'autres peuples slaves. Une des plus belles parmi ces formes, le sonnet (une forme préférée par quelques-uns des plus grands poètes modernes depuis le treizième siècle, comme Dante, Pétrarque, Le Tasse, Lope de Vega, Camoes, Shakespeare, Milton etc. et introduite en Allemagne par les poètes de l'école silésienne, comme Opitz, Fleming, etc. renouvelée par Bürger et Schlegel et depuis souvent retravaillée par d'autres [...]) a été maniée par le premier poète polonais, A. Mickiewicz, avec tout son art [...]. Parmi les sonnets de Prešeren, plusieurs, notamment ceux contenus dans $K r$. Zhb. II. str. 24, III. str. 29 nous paraissent être, par leur forme, de vrais modèles du genre». 
Les propositions de Čop ne se limitent pas aux aspects formels de la poésie, mais portent aussi sur le contenu et la langue littéraire. Un article publié dans l'Illyrisches Blatt illustre clairement son jugement sur la situation culturelle de son époque ainsi que sa vision de la littérature:

Unserer (größtentheil jansenistischen) Geistlichkeit, die doch das vorzüglichste krainische Lesepublikum ausmacht, scheint jede Art von Schriftstellerey, die nicht auf die Bedürfnisse des Landmannes (und zwar vor allem auf die geistlichen) berechnet ist, wenigstens überflüßig, erotische Poesie aber höchst sündhaft. Diese Leute glauben, eine Sprache sey hinlänglich gebildet, wenn man Katechismus und Gebethbuch in derselben schreiben kann; und wenn sie vollends ein Dutzend Grammatiken besitzt, dann bleibe nichts zu wünschen übrig. Wie es um die ästhetische Bildung dieser Leute steht, können Sie daraus schließen, daß ich mich veranlaßt gesehen, denselben ein langes Gerede über die südlichen poet. Formen etc. zu machen. ${ }^{11}$

Il existe malheureusement peu de documents témoignant d'une communication écrite sur des questions littéraires entre Prešeren et Čop, mais on peut supposer qu'ils communiquaient de vive voix, et fréquemment, étant donné qu'ils étaient amis et vivaient dans la même ville ${ }^{12}$. L'influence des idées de Čop et des auteurs qu'il préconise est évidente dans le choix que Prešeren fait des formes poétiques, tout comme dans ses efforts pour perfectionner la langue littéraire ${ }^{13}$. Le sonnet, par exemple, a été introduit dans la littérature slovène par Prešeren, sans doute encouragé en cela par Čop. S'il est vrai que Prešeren n'est pas l'auteur du tout premier sonnet slovène (écrit par Koseski en 1818), il reste néanmoins le fondateur du sonnet en langue slovène et le créateur d'un canon qui acquerra valeur de norme pour tout auteur de sonnets en slovène ${ }^{14}$.

11. «Notre clergé (majoritairement janseniste), qui constitue l'élite du public de lecteurs en Slovénie, trouve toute forme de littérature qui n'est pas conçue pour plaire aux provinciaux, et surtout au clergé, au mieux superflue, et considère la poésie d'amour comme un grand péché. Ces gens pensent qu'une langue est suffisamment développée du moment que l'on peut rédiger dans cette langue le catéchisme et des livres de prière, et si avec ça elle possède également une douzaine de grammaires, alors pour eux tout est parfait. Quant à la culture esthétique de ces gens, il vous suffit de savoir que j'ai cru nécessaire de leur faire un long discours sur les formes poétiques méridionales etc.» (Lettre à František Čelakovskỷ datée du 14 mars 1833, in Matija Čop, Pisma Matija Čopa, I, ed. Anton Slodnjak et Janko Kos, Ljubljana, SAZU, 1986, p. 242).

12. Voir France PREŠEREN, Pisma: ob dvestoti obletnici pesnikovega rojstva: 3. decembra 1800 3. decembra 2000, ed. Jože Kastelic, Ljubljana, Rokus, 2000, p. 39.

13. Sur le rôle de Matija Čop dans la formation poétique de Prešeren voir aussi Janko Kos, "Problem Čopovega posredništva», in IDEM, Prešeren in evropska romantika, Ljubljana, DZS, 1970, p. 38-54.

14. Boris A. NOVAK, «La corona di sonetti di Prešeren: per la storia di una forma poetica», Ricerche slavistiche, 47, 2003, p. 49. 
Comme nous l'avons vu, on ne peut guère parler d'un pétrarquisme slovène comme mouvement ou tendance de grande portée. La réception par Prešeren de la poésie de Pétrarque ne dépend pas d'un courant, comme elle ne crée pas de courant proprement dit. Néanmoins, en raison de la place centrale occupée par cet auteur dans la littérature slovène, son "pétrarquisme» connaîtra une vaste diffusion dans l'espace culturel slovène.

Les réminiscences pétrarquistes dans la poésie de Prešeren ont été minutieusement analysées par de nombreux slavistes, couvrant toute une gamme d'aspects thématiques et stylistiques qu'il serait évidemment impossible de résumer $\mathrm{ici}^{15}$. Un aspect, qui nous semble particulièrement révélateur, concerne les évocations explicites de Pétrarque dans les poèmes de Prešeren; par leur fréquence et leur force expressive elles témoignent de l'importance que Pétrarque revêtait pour Prešeren; il nous faut réfléchir à la fonction spécifique de ces renvois à Pétrarque qui semblent bien être un élément constitutif de l'œuvre du poète slovène. On rencontre Pétrarque et son œuvre, chez Prešeren, essentiellement sous quatre formes:

- directement, c'est à dire par la mention de son nom (mais Pétrarque n'est jamais interpellé à la deuxième personne en tant qu'interlocuteur du sujet lyrique) ${ }^{16}$;

15. Voir, par exemple, Pavel GrošELJ, «Prešeren in Petrarka», cit. ; Bartolomeo CALVI, Fonti italiane e latine nel Prešeren maggiore, Turin, Società Editrice Internazionale, 1959; Boris Paternu, France Prešeren in njegovo pesniško delo, I, cit.; Anton SlODNJAK, "Petrarca in Prešeren", in Petrarca i petrarkizam u slavenskim zemljama. Radovi Međunarodnog simpozija I Petrarca e il petrarchismo nei paesi slavi. Atti del convegno internazionale, cit., p. 469-474; Jože POGAČNIK, «Slovenski romantizam i Petrarca», cit.; Janko Kos, Primerjalna zgodovina slovenske literature, cit., p. 88-91; Vid SNOJ, «Ženska sonce: razris figure iz Petrarcovega in Prešernovega pesništva» ("Die Frau als Sonne: eine Analyse der Figur in Petrarcas und Prešerens Dichtung»), in France Prešeren - kultura - Evropa, ed. Jože Faganel, Ljubljana, ZRC SAZU, 2002, p. 61-73; Boris PATERNU, «L'antipetrarchismo di Prešeren», Ricerche slavistiche, 47, 2003, p. 9-22. En effet, Pétrarque a été étudié par les Slovènes surtout en parallèle avec Prešeren, à l'exception de quelques essais introductifs aux éditions de ses poèmes (Stanko ŠKERLJ, «Uvod», in Francesco PETRARCA, Soneti in kancone, cit., p. V-XXXIX; Atilij RAKAR, "Rerum vulgarium fragmenta", in Francesco PetrarCA, Stihi, trad. par Andrej Capuder, Ljubljana, Mladinska knjiga, 1980, p. 158-190; Andrej CAPUder, "Petrarka, poslednji trubadur», in Francesco Petrarca, Francesco Petrarca, trad. par A. Capuder, Ljubljana, Mladinska knjiga, 1987; Andrej CAPUdER, «Francesco Petrarca včeraj, danes", in Francesco PetrarCA, Soneti, trad. par A. Capuder, Ljubljana, Mihelač, 1995, p. 322-328.

16. Il primo amore (Prva ljubezen), Glossa (Glosa), Sonetto d'amore V (Ljubeznjeni sonet V), Noi due sognai nel santo paradiso (Sanjalo se mi je, de v svetem raji). 
- indirectement, par des allusions transparentes qui renvoient à Pétrarque sans toutefois mentionner son nom, en rappelant, par exemple, la date de sa rencontre avec Laure ${ }^{17}$;

- par des éléments se référant à Laure ${ }^{18}$;

- par la citation directe de ses vers ${ }^{19}$.

Laure est évoquée de manière explicite dans la poésie de Prešeren comme terme de comparaison de sa dame; parfois il en cite le nom ("più che di Corinna, Delia, Cinzia, o Monna Laura / danno recherebbe l'obliare il tuo nome ${ }^{20}$ ) parfois il a recours à une paraphrase («quella che a Petrarca accese il cuore / l'estremo venerdì quaresimale » ${ }^{21}$; à ce dernier exemple est étroitement lié Correva l'anno mille ed ottocento).

Mais c'est aux mentions explicites de Pétrarque que nous allons nous intéresser de plus près, car elles ont une fonction spécifique dans le discours de Prešeren sur la poésie, notamment dans ses réflexions sur le rôle $\mathrm{du}$ poète et sur l'autonomie de la poésie. Nous proposons d'esquisser un parcours idéal (qui ne coïncide pas nécessairement avec un parcours chronologique) en trois étapes, représentées par Glossa, Sonetto d'amore $V$, et Noi due sognai nel santo paradiso.

Dans Glossa, Prešeren déplore la position sociale défavorable du poète et critique le manque d'intérêt pour la littérature de la part du public slovène. Il énumère une série d'auteurs (reproduisant en partie la liste des auteurs recommandés par Čop) qui représentent les classiques de la littérature européenne, donc la littérature par excellence (voir p. suiv.).

Pétrarque figure parmi les auteurs classiques garants de la haute valeur de la littérature. Une telle vision de la littérature oppose le poète et les richesses matérielles, selon une conception romantique de la supériorité inhérente du poète que Prešeren, à ce moment, semble embrasser avec ardeur.

17. Correva l'anno mille ed ottocento (Je od vesel'ga časa teklo leto).

18. Il primo amore, Gazzella I (Gazela I), Noi due sognai nel santo paradiso.

19. Dans la première version de Il primo amore, publiée dans L'Abeille carniolienne, Prešeren inclut en note la citation: «Era 'l giorno, ch'al sol si scoloraro / Per la pietà del suo fattore i rai, / Quand'i' fui preso ecc.» (Krajnska čbelica, 1832 (3), na svitlobo dal M. Kastelic, reproduction anastatique de la première édition, Ljubljana, Mladinska knjiga, 1969, p. 19). La citation ne figure pas dans la version définitive des Poèmes

20. Gazzella I, in Poesie, trad. par Francesco Husu, cit., p. 99; «bolj ko Délije, Korine, Cintije al'Lavre / bi bilo pozabit' škoda tvojega imena" (Gazela I, in Poezije doktorja Franceta Prešerna, cit., p. 149).

21. Il primo amore, in Poesie, trad. par Francesco Husu, cit., p. 90 ; «ki je od nje na zadnji petek v posti / Petrarkovo biló srce užgano" (Prva ljubezen, in Poezije doktorja Franceta Prešerna, cit., p. 134). 
IRENA PROSENC ŠEGULA

Glossa

"Cieco è l'inventor di versi, ne trae burla il Carniolano; non ha fortuna il poeta, vive egli e muore in povertà."

Vediamo per primo Omero, vecchio, misero e mendico; nel gelido Ponto, Ovidio; storie uguali son per gli altri: Dante è l'esempio di quale sorte colpisca i poeti ; lo confermano scrittori quali Camões e Cervantes, quanta fatica al Parnaso cieco è l'inventor di versi.

A che servono i poeti? a che un Petrarca o un Tasso? Non da oggi se lo chiedon certe teste vuote e dure.

Chi più ascolta il dolce canto che fa vivere Matjaž, le battaglie dei Croati, chi il poeta dell'Illiria chi gli sciami dell' "Ape»? ne trae burla il Carniolano.

Ieri appena un imbroglione rifilava vecchie cose, lino e nastro misurava, ora invece ha già un castello. Vada in Cina e oltre il poeta, dove s'apron nuovi mondi, macchi pur le man d'inchiostro, di svegliar l'amor sperando d'una bella e giovin donna, fortuna non ha il poeta.

Di cantar però non cessa ; ammucchiate soldi certi, fate pur vostri i castelli, senza pensier godeteli! L'orizzonte intero è il castel senza custode al poeta, gli ori in esso son le aurore le rugiade son gli argenti, questi son i suoi soli averi vive egli e muore in povertà. ${ }^{22}$
Glosa

"Slep je, kdor se spetjem ukvarja, Kranjec moj mu osle kaže; реvсu vedno sreča laže, on živi, umrjè brez d'narja."

Le začniva pri Homêri, prosil reva dni je stare; mraz Ovid'ja v Pontu tare; drugih pevcev zgodbe beri; nam spričuje Alighiêri, káko sreča pevce udarja; nam spričujeta pisarja Luzijade, Don Kihota, kákošna Parnasa pota, slep je, kdor se s petjem ukvarja.

Kaj Petrarkov, kaj nam Tassov treba pevcev je prijetnih? Slišim od butic neukretnih prašat'zdanjih, prednjih časov. Kómur mar prijetnih glasov pesmi, ki pojo Matjaže, boje krog hrvaške straže, mar, kar pevec pel Ilir'je, mar «Čebel'ce» roji štirje, Kranjec moj mu osle kaže.

Lani je slepar staríno še prodajal, nosil škatle, meril platno, trak na vatle, letos kupi si graščino.

Naj gre pevec v daljno Kino, še naprej se pot mu kaže, naj si s tinto prste maže, naj ljubezen si obeta vneti lepega dekleta, pevcu vedno sreča laže.

Vèndar peti on ne jenja; grab'te d'narje vkup gotove, kupovájte si gradóve,

v njih živíte brez trpljenja! Kóder se nebó razpenja, grad je pevca brez vratarja, $\mathrm{v}$ njem zlatnina čista zarja, srebrnina rosa trave, $s$ tem posestvom brez težave on živi, umrjè brez d'narja. ${ }^{23}$

22. Poesie, trad. par Giorgio Depangher, cit., p. 15.

23. Poezije doktorja Franceta Prešerna, cit., p. 138. 
Dans le Sonetto d'amore $V$, cependant, Pétrarque assume une fonction diamétralement opposée: il y est le "pauvre» poète, (plus spécifiquement: poète d'amour), que le sujet lyrique répudie d'emblée, optant pour les richesses matérielles qu'il avait jadis refusées:

Sonetto d'amore $V$

Cupido! tu e la tua bella vecchia, più non mi prenderete per il naso; gratis non canterò le vostre lodi per sempre, come il povero Petrarca.

Molti anni la Parca ha già filato; l'estro dei versi cosa mi ha portato? Ancor nessuna donna mi ha voluto, più non v'incenserò senza pensare.

Vuoti scherzi son le parole vostre; mi sento stanco di subirvi, ingrate; questi anni che ancor mi son rimasti,

dalle liti tutto il giorno oro trarrò, coppe vuoterò a sera con amici, nubi d'affanni col vino scaccerò. ${ }^{24}$
Ljubeznjeni sonet $V$

\author{
Kupído, ti in tvoja lepa starka \\ ne bosta dalje me za nos vodila; \\ ne bom pel vaj'ne hvale brez plačila \\ do konca dni ko siromak Petrarka. \\ Dovolj mi let je že napredla Parka; \\ kogá mi je prinesla pevska žila? \\ Nobena me še ni deklet ljubíla, \\ kadíl ne bom več vaju brez preudarka. \\ Obéti vaj'ni so le prazne šale; \\ sit, nehvaležnika, sem vaj'ne tlake; \\ te leta, ki so meni še ostale, \\ cel dan iz pravd koval bom rumenjake, \\ zvečer $s$ prijat'lji praznil bom bokale, \\ preganjal z vinom bom skrbi oblake. ${ }^{25}$
}

L'épithète "pauvre» n'est d'ailleurs nullement réservée à Pétrarque; bien au contraire, elle est appliquée par Prešeren à lui-même. Elle est utilisée dans toute une gamme de variations: "pauvre», "misérable», "pitoyable» («siromak», «revež»), pour décrire le sujet lyrique dans une double dimension: celle du poète (nous retrouvons donc encore une fois la réflexion sur le rôle et la position sociale du poète), et celle de l'homme souffrant à cause de l'amour (dans la poésie de Prešeren, en effet, l'amour n'est jamais partagé, à l'instar de l'amour pétrarquiste).

Pour Prešeren le côté matériel de la vie s'oppose donc tant à l'amour qu'à la poésie même. Pourtant, ses doutes sont loin de celles exprimées par Pétrarque; celui-ci, en effet, s'inquiète de ne pas pouvoir s'exprimer ("I mei gravi sospir'non vanno in rime, / e 'l mio duro martir vince ogni stile») ${ }^{26}$ et de ne pas être à la hauteur de son sujet («le mie basse rime», "le mie roche rime», "le mie stanche rime»), conscient de la mutatio

s24. Poesie, trad. par Giorgio Depangher, cit., p. 45.

25. Poezije doktorja Franceta Prešerna, cit., p. 163.

26. Canzoniere 332, 11-12 (cité d'après Francesco Petrarca, Canzoniere, a cura di M. Santagata, Milano, Mondadori, 1996). 
animae $^{27}$ qui a influencé son style ("Ove è condutto il mio amoroso stile?", «io vo col penser cangiando stile», "l mutato stile») ${ }^{28}$. Prešeren, pour sa part, se montre toujours sûr de sa maîtrise poétique. En revanche, sa réflexion poétique se focalise sur la question fondamentale de la mission du poète et revendique l'autonomie de la poésie face à la situation culturelle de son époque, défavorable à la poésie.

Plusieurs critiques ont interprété ce Sonetto d'amore comme un refus (temporaire) de Pétrarque de la part de Prešeren, faisant partie du mouvement continu de ses rapprochements et éloignements du pétrarquisme ${ }^{29}$. Néanmoins, Prešeren semble finalement se réconcilier avec Pétrarque (ou bien avec son propre rôle de poète d'amour) dans Noi due sognai nel santo paradiso. Il reconnaît la supériorité poétique de Pétrarque, mais revendique pour sa dame la même dignité qui distingue Laure et exalte son pouvoir d'ennoblir la poésie :

Noi due sognai nel santo paradiso felici come non si può pensare: m'era lontano il tempo della vita, quando età e luoghi avevan noi diviso.

Da sorella sedevi accanto a Laura, rivisitando insieme i giorni andati e conversando in quel soave idioma, come fosse per voi prova d'onore.

Sulla bilancia, lì, di San Michele, messi a confronto i versi con Petrarca, il piatto mio fu spinto verso l'alto.

Ma aggiunte le virtù delle due donne ciascuna ai propri versi, il piatto suo non andò verso il basso più del mio. ${ }^{30}$

\author{
Sanjálo se mi je, da v svetem raji \\ bilà sva srečna tam brez zapopádka: \\ bilá je preč življenja doba kratka, \\ kjer me od tebe ločjo časi, kraji. \\ Sedela z Lavro ti si sestra mlaji, \\ pred vama je bilà dni prejžnjih prat'ka, \\ bilà med vama govorica sladka, \\ kakó slovela k'tera je od vaji. \\ In tam na tehtnico svet'ga Mihela \\ s Petrárkom d'jala sva sonete svoje, \\ visoko moja skled'ca je zletela. \\ Prid'jala čednosti sva nje in tvoje \\ vsak svojim pesmam, in skodela \\ njegà bilà ni niž od skled'ce moje. ${ }^{31}$
}

27. Voir Roberto ANTONelli, "Rerum vulgarium fragmenta di Francesco Petrarca», in Letteratura italiana. Le opere, I: Dalle origini al Cinquecento, ed. Alberto Asor Rosa, Turin, Einaudi, 1992, p. 377-471

28. Canzoniere 332,$13 ; 332,28 ; 332,64$.

29. Voir, par exemple, Boris PATERnU, «L'antipetrarchismo di Prešeren », cit.

30. Poesie, trad. par Giorgio Depangher,. cit., p. 77.

31. Poezije doktorja Franceta Prešerna, cit., p. 181. 
Les poèmes dans lesquels Pétrarque est explicitement nommé ne sont qu'un fragment de la très riche réflexion de Prešeren sur les fonctions de la poésie et du poète. Dans d'autres poèmes, Prešeren approfondit sa vision du poète, suspendu entre la misère et la perfection ${ }^{32}$, ainsi que l'idée de la dame ayant un rôle actif, voire décisif dans la création poétique ${ }^{33}$.

L'influence de Pétrarque se présente dans la poésie de Prešeren sous des aspects multiples, allant du choix même d'introduire le sonnet dans la littérature slovène, jusqu'à la reprise de nombreux éléments thématiques typiques de la poésie de Pétrarque (surtout des motifs liés à la genèse de l'amour, à la figure de la dame, aux effets de l'amour sur le sujet lyrique). La thèse que ces éléments ne soient pas simplement imités par Prešeren, mais consciemment élaborés et assimilés dans un contexte qui lui est propre, est largement acceptée par la critique; il est par ailleurs indéniable que le répertoire des motifs de la poésie d'amour chez Prešeren ne se limite aucunement à la conception pétrarquiste. Cette prise de position consciente de la part de Prešeren se manifeste aussi dans l'usage qu'il fait des références à Pétrarque et à son œuvre; elles sont en effet un élément central de sa réflexion poétique, et Pétrarque devient l'un des appuis sur lesquels Prešeren construit sa vision de la poésie.

32. Voir In ricordo di Valentino Vodnik (Vspomin Valentina Vodnika), Al poeta (Pevcu), Il giullare (Orglar), Sonetto d'amore I (Sonet ljubezni I), ainsi que quelques poèmes en allemand: Obschon die Lieder aus dem Vaterlande, Ihr, die entsprossen aus dem Slawenstamme, Warum sie, wert, daß Sänger aller Zungen, Nichts trägt an ihm des Dichtergeists Gepräge, An eine junge Dichterin (in France PrešERen, Poezije in pisma, ed. Janko Kos, Ljubljana, Mladinska knjiga, 1998).

33. Serto di sonetti (Sonetni venec). 